\title{
The protective role of mesencephalic astrocyte-derived neurotrophic factor in endoplasmic reticulum stress in RT4-D6P2T schwannoma sells with the S63del MPZ mutation
}

\author{
Bo Sun ${ }^{1,2}$, Hongfen Wang ${ }^{1}$, Yanran $\mathrm{Li}^{1}$, Zhengqing $\mathrm{He}^{1}$, Fang Cui ${ }^{1}$, Fei Yang ${ }^{1}$, Xusheng Huang ${ }^{1, *}$ \\ ${ }^{1}$ Neurological Department of the First Medical Center, Chinese PLA General Hospital, 100853 Beijing, China, ${ }^{2}$ Geriatric Neurological Department of \\ the Second Medical Center \& National Clinical Research Center for Geriatric Diseases, Chinese PLA General Hospital, 100853 Beijing, China \\ *Correspondence: lewishhuang301@163.com (Xusheng Huang) \\ Academic Editor: Graham Pawelec \\ Submitted: 18 September 2021 Revised: 16 November 2021 Accepted: 26 November 2021 Published: 4 January 2022
}

\begin{abstract}
Background: Endoplasmic reticulum stress (ERS) occurred in S63del mutant CMT1B mice model, and few drugs has been studied. Mesencephalic astrocyte-derived neurotrophic factor (MANF) can inhibit ERS. This study aimed at investigating the effect of MANF on ERS of RT4-D6P2T schwannoma cells with S63del MPZ Mutation. Methods: Experimental grouping: blank control group, blank control + MANF group, lentivirus group, lentivirus + MANF group, S63del MPZ group, S63del MPZ + MANF group. CCK8 and Annexin-FITC/PI were used to detect cell proliferation and apoptosis. JC-1 was used to detect $\Delta \Psi \mathrm{m}$. MANF, GRP78 and CHOP mRNA and protein were detected by using RT-qPCR, western blotting and immunofluorescence. ER-Tracker and mito-tracker were used to observe the morphology of endoplasmic reticulum (ER) and mitochondria. Results: Cell proliferation decreased $(p<0.001)$ and apoptosis increased $(p<0.001)$ in S63del MPZ group; cell proliferation increased $(p=0.005)$ and apoptosis decreased $(p<0.001)$ in S63del MPZ + MANF group. $\Delta \Psi \mathrm{m}$ decreased $(p<0.001)$, MANF, GRP78, CHOP, ATF6, P-PERK/PERK, P-IRE1/IRE1, Bax and Caspase3 increased $(p<0.001)$ and Bcl2 decreased $(p<0.001)$ in S63del MPZ group. MANF, GRP78, CHOP, ATF6, P-PERK/PERK, P-IRE1/IRE1, Bax and Caspase3 decreased $(p<0.001)$ and Bcl2 increased $(p<0.001)$ in S63del MPZ group. Conclusions: ERS occurred in RT4-D6P2T cells with S63del MPZ mutation, and MANF exerted protective effect in RT4-D6P2T cells with S63del MPZ mutation.
\end{abstract}

Keywords: Charcot-Marie-Tooth disease type 1B; Endoplasmic reticulum stress; Unfolded protein response; Mesencephalic astrocytederived neurotrophic factor; RT4-D6P2T schwannoma cell

\section{Introduction}

Charcot-Marie-Tooth disease (CMT) is the most common hereditary peripheral neuropathy [1]. More than 90 genes have been reported to be associated with CMT [2]. Mutations in PMP22, MPZ, GJB1 and MFN account for over $80 \%$ of the mutations in this disease [1]. CMT1B, caused by myelin protein zero (MPZ) mutations, is the second most common type of CMT1 [3,4]. More than 200 MPZ mutations have been detected [5], of which the S63del and R98C MPZ mutations have been used to study the pathogenesis of CMT1B [6-8].

Schwann cells are the myelinating glia of the peripheral nervous system and produce an enormous amount of proteins, among which MPZ accounts for 20\% [6]. Deletion of Ser63 in MPZ (P0S63del) causes CMT1B. In patients and mice with the S63del MPZ mutation, the P0S63del protein is retained in the endoplasmic reticulum (ER) and triggers the unfolded protein response (UPR) [7,9], leading to endoplasmic reticulum stress (ERS). The UPR maintains cellular homeostasis by reducing the amount of aberrant proteins accumulated in the ER [10,11]. However, when it becomes persistent, the UPR activates maladaptive effectors involved in cell death and dedifferentiation [9,12]. The UPR pathways involve the molecules inositol-requiring protein-1 (IRE1), activating transcription factor-6 (ATF6), and protein kinase RNA-like ER kinase (PERK) [7]. Upon activation, IRE1 mediates the splicing of X-box binding protein 1 (Xbp1) mRNA [13-15]. $\mathrm{XBP} 1 \mathrm{~s}$ reprograms the transcription of genes involved in protein folding, degradation, and ER biogenesis [16-18]. The ATF6 pathway promotes the expression of ER-resident chaperones [19]. The kinase PERK phosphorylates eukaryotic translation initiation factor $2 \alpha(\mathrm{eIF} 2 \alpha)$ on Ser51, which causes a global attenuation in protein translation and thereby limits the accumulation of client proteins in the ER [20]. PERK or CHOP ablation attenuates the phenotype of a mouse model with the S63del MPZ mutation [6,7]. Sephin1 prevented the molecular, morphological, and motor defects of the S63del MPZ mice [21]. Curcumin derivatives promote Schwann cell differentiation and improve neuropathy in R98C CMT1B mice [8].

Mesencephalic astrocyte-derived neurotrophic factor (MANF) is an evolutionarily conserved neurotrophic factor that was first discovered to have protective effects on dopaminergic neurons in the embryonic mesencephalon 

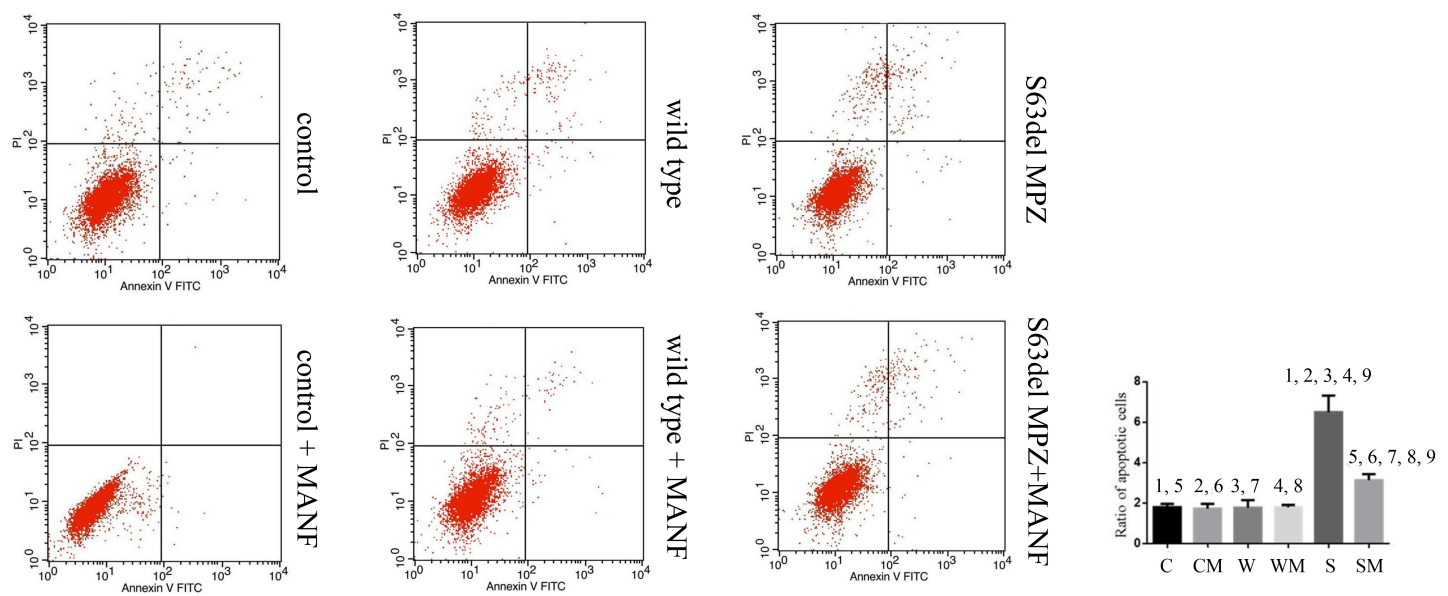

Fig. 1. Flow cytometry: Annexin V-FITC/PI double staining. C: control, CM: control + MANF, W: wild type, WM: wild type + MANF, S: S63del MPZ, and SM: S63del MPZ + MANF. 1, 2, 3, 4, 5, 6, 7, 8, 9: The difference between two groups was statistically significant $(p<0.05) ; \mathrm{n}=3$.

in vitro [22]. MANF inhibits the UPR and modulates the transcription of genes induced by ERS, including the HSP70 family, the ATF family, xbp-1 and MARK [2327]. 6-OHDA can lead to ERS in SHSY-5Y cells; however, when MANF intervention is applied, transcriptomics shows downregulation of the transcription of genes associated with ERS and cell apoptosis [28]. In addition, MANF plays protective roles in diabetes, renal disease and Parkinson's disease [29-31].

In this study, we found that ERS occurred in RT4D6P2T schwannoma cells with the S63del MPZ mutation and MANF played a protective role against ERS associated RT4-D6P2T schwannoma cells with the S63del MPZ mutation.

\section{Methods}

\subsection{The RT4-D6P2T schwannoma cell culture and transfection}

The RT4-D6P2T schwannoma cell line was purchased from American Type Culture Collection (ATCC). Briefly, cells were cultured continuously in high-glucose DMEM supplemented with $10 \% \mathrm{FBS}$ and maintained at $37{ }^{\circ} \mathrm{C}$ and $5 \% \mathrm{CO}_{2}$. pLVX-MPZ (Sdel63)-PGK-Puro and pLVXPGK-Puro plasmids were constructed and packaged into lentiviral vectors. RT4-D6P2T schwannoma cells were plated in 6-well round-bottom plates. After 48 hours, the cells were incubated with culture medium supplemented with $6 \mu \mathrm{g} / \mathrm{mL}$ puromycin to select a stably transfected cell line, which was assessed by using RT-qPCR and Western blotting.

\subsection{Treatment of RT4-D6P2T schwannoma cells with $M A N F$}

RT4-D6P2T schwannoma cells were treated as follows: the control, control + MANF, pLVX-MPZ-PGK-
Puro (wild type), pLVX-MPZ-PGK-Puro + MANF (wild type + MANF), pLVX-MPZ (Sdel63)-PGK-Puro (S63del MPZ) and pLVX-MPZ (Sdel63)-PGK-Puro+MANF (S63del MPZ + MANF) groups. MANF intervention (100 nM) lasted for 24 hours.

\subsection{Cell counting kit-8 (CCK-8) assay for cell viability and toxicity}

For assessing the cell viability and toxicity of RT4D6P2T schwannoma cells, a CCK-8 assay was performed. RT4-D6P2T schwannoma cells with above different treatments were cultured for 24 hours and subjected to the CCK8 assay. Cell viability was assessed with CCK-8 (Solarbio, China) according to the manufacturer's instructions.

\subsection{Induction of cell apoptosis assay}

RT4-D6P2T schwannoma cells $\left(1 \times 10^{6}\right.$ cells/well $)$ were plated in six-well plates for the apoptosis assay. The cells were harvested and treated with an Annexin VFITC/PI apoptosis detection kit based on the manufacturer's protocol. Afterwards, the cells were evaluated with a BD FACSCalibur using BD CellQuest Pro software for analysis.

\subsection{Mitochondrial membrane potential $(\Delta \Psi \mathrm{\Psi})$ detection}

The $\Delta \Psi \mathrm{m}$ was analyzed using JC-1 staining (Beyotime Institute of Biotechnology). Briefly, cells were washed with ice-cold PBS and then stained with $2.5 \mathrm{~g} / \mathrm{mL}$ JC-1 for $30 \mathrm{~min}$ at $37^{\circ} \mathrm{C}$. After being washed with binding buffer, the cells were analyzed by using fluorescence microscopy. The fluorescence intensity was detected by Image J software (v1.8.0, National Institutes of Health, Bethesda, MD, USA), and then the ratio of red to green fluorescence intensity was calculated. The results are presented as the relative aggregate-to-monomer (red/green) fluorescence intensity ratio. 

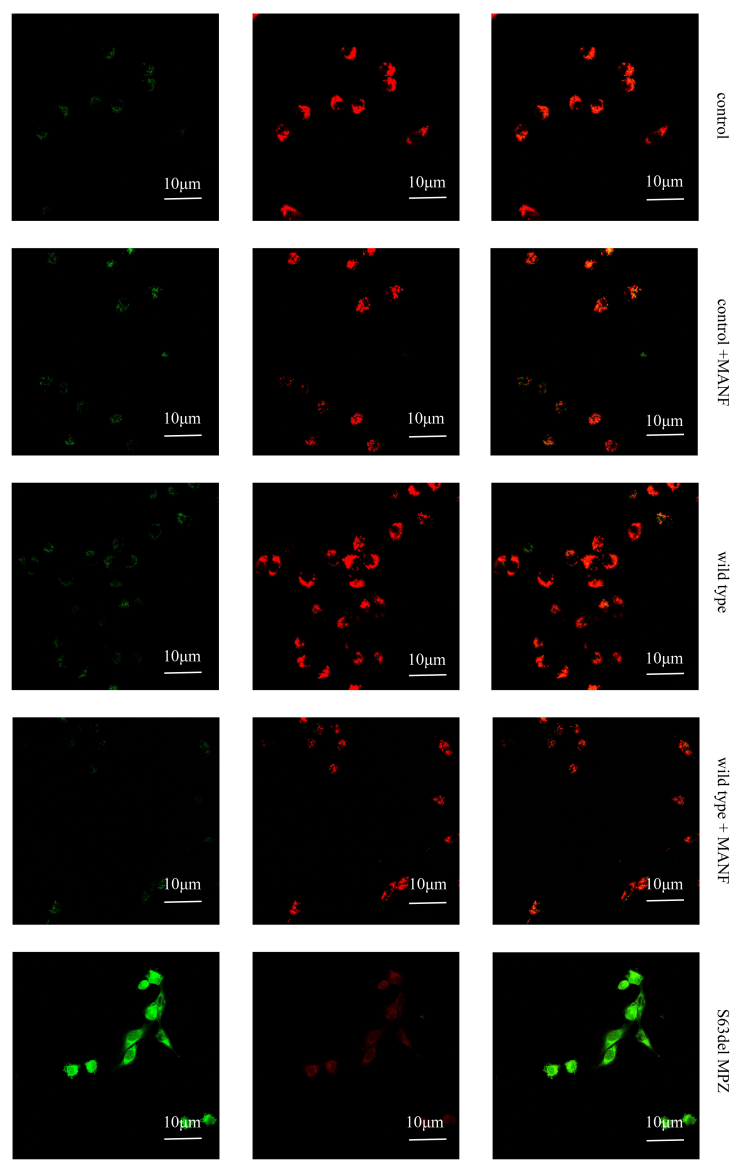

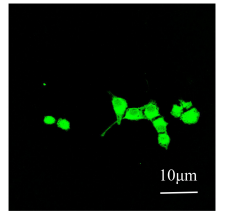

Monomer

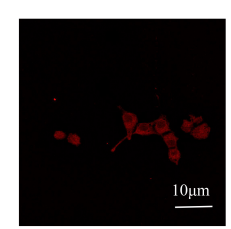

Polymer

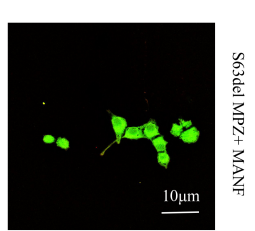

Merge

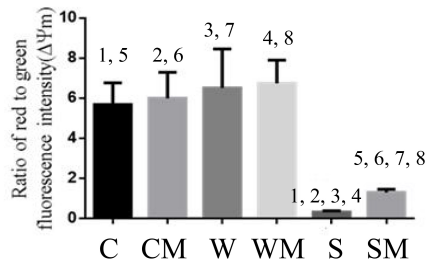

C CM W WM S SM

Fig. 2. Detection of the mitochondrial membrane potential through confocal microscopy. C: control, CM: control + MANF, W: wild type, WM: wild type + MANF, S: S63del MPZ, and SM: S63del MPZ + MANF. 1, 2, 3, 4, 5, 6, 7, 8: The difference between two groups was statistically significant $(p<0.001) ; \mathrm{n}=3$.

RT4-D6P2T schwannoma cells $\left(1 \times 10^{6}\right.$ cells/well $)$ were plated in six-well plates for $\Delta \Psi$ m detection. The cells were harvested and treated with a JC-1 kit according to the manufacturer's protocol (Solarbio, China). Afterwards, the cells were evaluated with a BD FACSCalibur using BD Cel1Quest Pro software for analysis.

\subsection{Quantitative polymerase chain reaction ( $q P C R$ )}

After MANF treatment for 24 hours, total RNA was extracted with TRIzol Reagent according to the manufacturer's recommended protocols. To generate cDNA, a total of $1 \mu \mathrm{g}$ RNA was used for reverse transcription using the TIANGEN RT Reagent Kit.

QPCR was performed on the Applied Biosystems 7500 RT-PCR System (Thermo Fisher Scientific) using Power SYBR Green PCR Master Mix (ABI) (Applied
Biosystems, Carlsbad, CA, USA). The $\beta$-actin gene was used as an endogenous control. The data were analyzed with the $2^{-\Delta \Delta C T}$ method. The primers were as follows:

MANF (F): 5'-ATGTGGACGCGCG-3', MANF (R): 5'-CTAGTCACACTCACCGTATTTTAGCTC$3^{\prime}$,

GRP78 (F): 5'-AGGATGTAGGCACGGTGGTT-3', GRP78 (R): 5'-GCCACATACGACGGTGTGAT-3', CHOP (F): 5'-CCTCGCTCTCCAGATTCCAG-3', CHOP (P): 5'-CCTTCTCCTTCATGCGCTGT-3', Actb (F): 5'-CACCATGTACCCAGGCATT-3', and Actb (R): 5'-ACTTGCGCTCAGGAGGAG-3' .

\subsection{Western blotting and immunofluorescence}

The following primary antibodies from Abcam, CST or Proteintech were used for Western blot analyses: anti- 

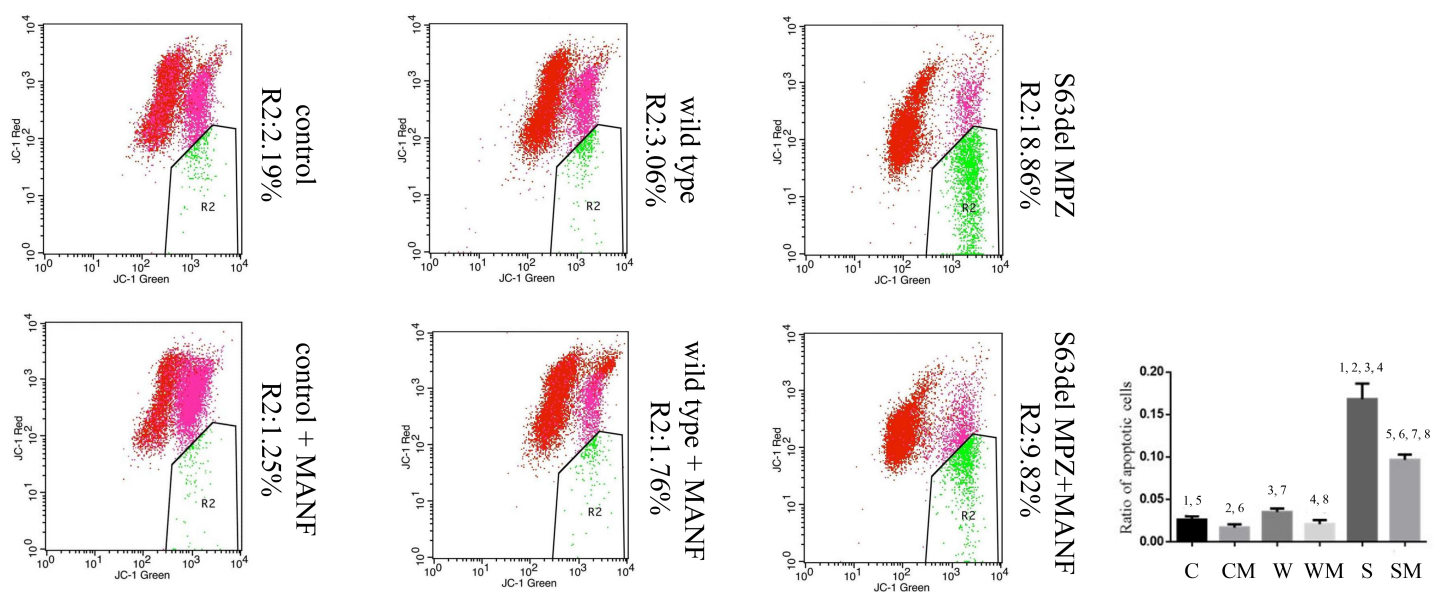

Fig. 3. Detection of the mitochondrial membrane potential through flow cytometry. C: control, CM: control + MANF, W: wild type, WM: wild type + MANF, S: S63del MPZ, and SM: S63del MPZ + MANF. 1, 2, 3, 4, 5, 6, 7, 8: The difference between two groups was statistically significant $(p<0.05) ; \mathrm{n}=3$.

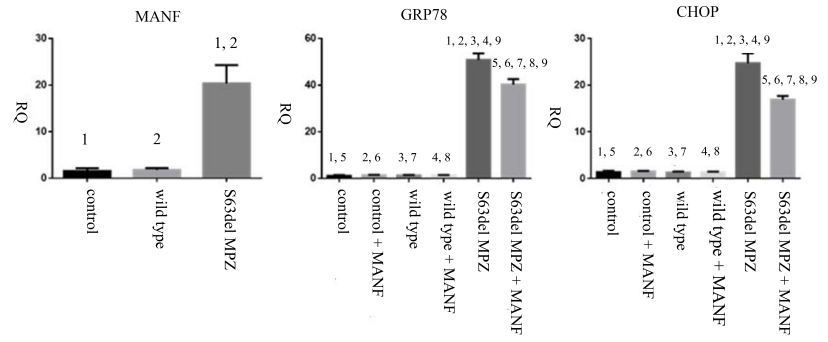

Fig. 4. MANF, GRP78, and CHOP relative mRNA expression. 1, 2, 3, 4, 5, 6, 7, 8, 9: The difference between two groups was statistically significant $(p<0.05) ; \mathrm{n}=3$.
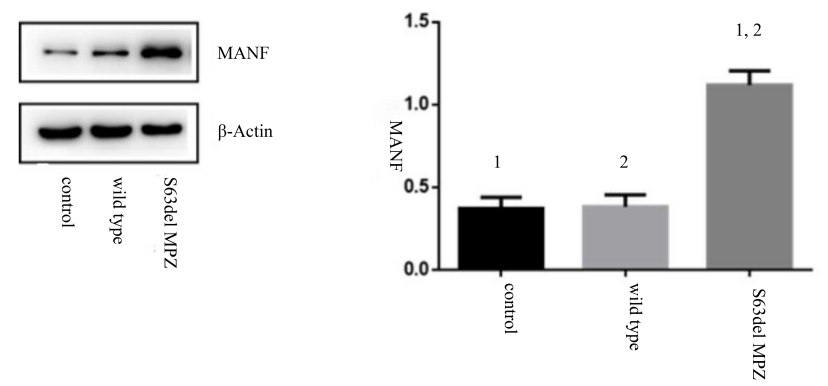

Fig. 5. MANF expression in each group. 1, 2: The difference between two groups was statistically significant $(p<0.001)$; $\mathrm{n}=$ 3 .

MANF (1-2 $\mu \mathrm{g} / \mathrm{mL})$, anti-GRP78 (1:1000), anti-CHOP (1:1000), anti-PERK (1:1000), anti-p-PERK (1:1000), antiIRE1 (1:1000), anti-p-IRE1 (1:1000), anti-ATF6 (1:1000), anti-Bcl2 (1:1000), anti-Bax (1:1000), anti-Caspase 3 (1:1000), and anti- $\beta$-actin $(1: 2000)$.

The following primary antibodies from Abcam, CST or Proteintech were used for immunofluorescence staining: anti-MANF (5 $\mu \mathrm{g} / \mathrm{mL})$, anti-GRP78 (1:100), anti-CHOP

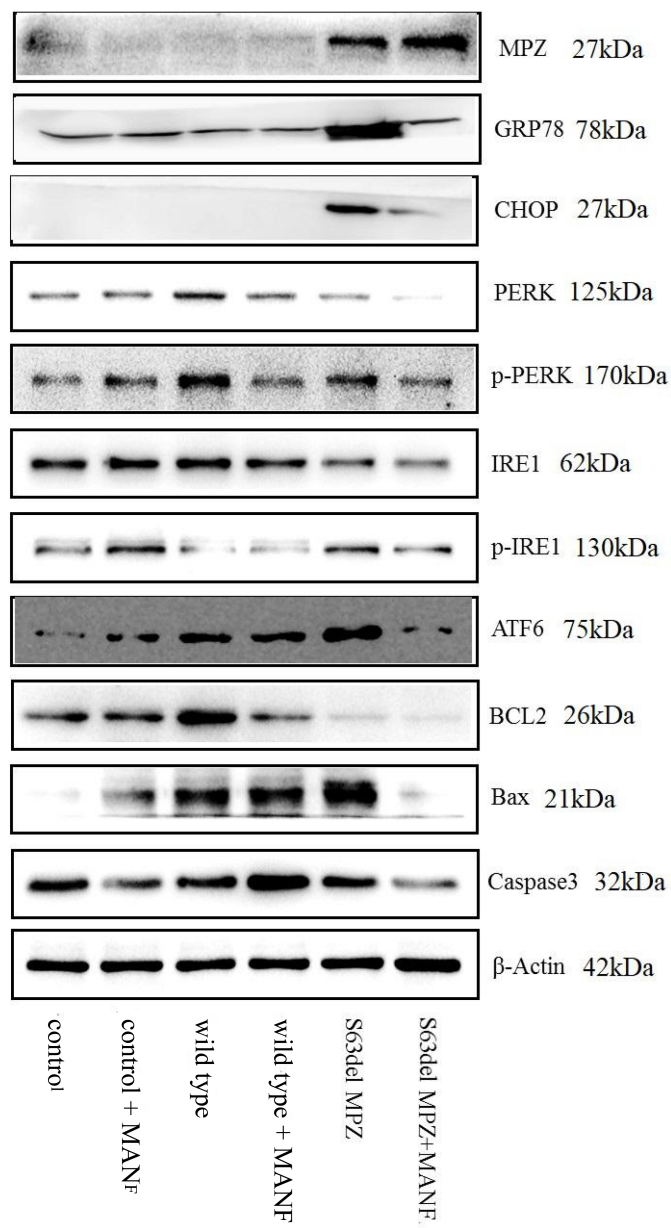

Fig. 6. MPZ, GRP78, CHOP, ATF6, P-PERK/PERK, PIRE1/IRE1, Bcl2, Bax, and Caspase3 expression in different groups.

(1:3200), anti-Bcl2 (1:100), anti-Bax (1:100), and antiCaspase 3 (1:100). 

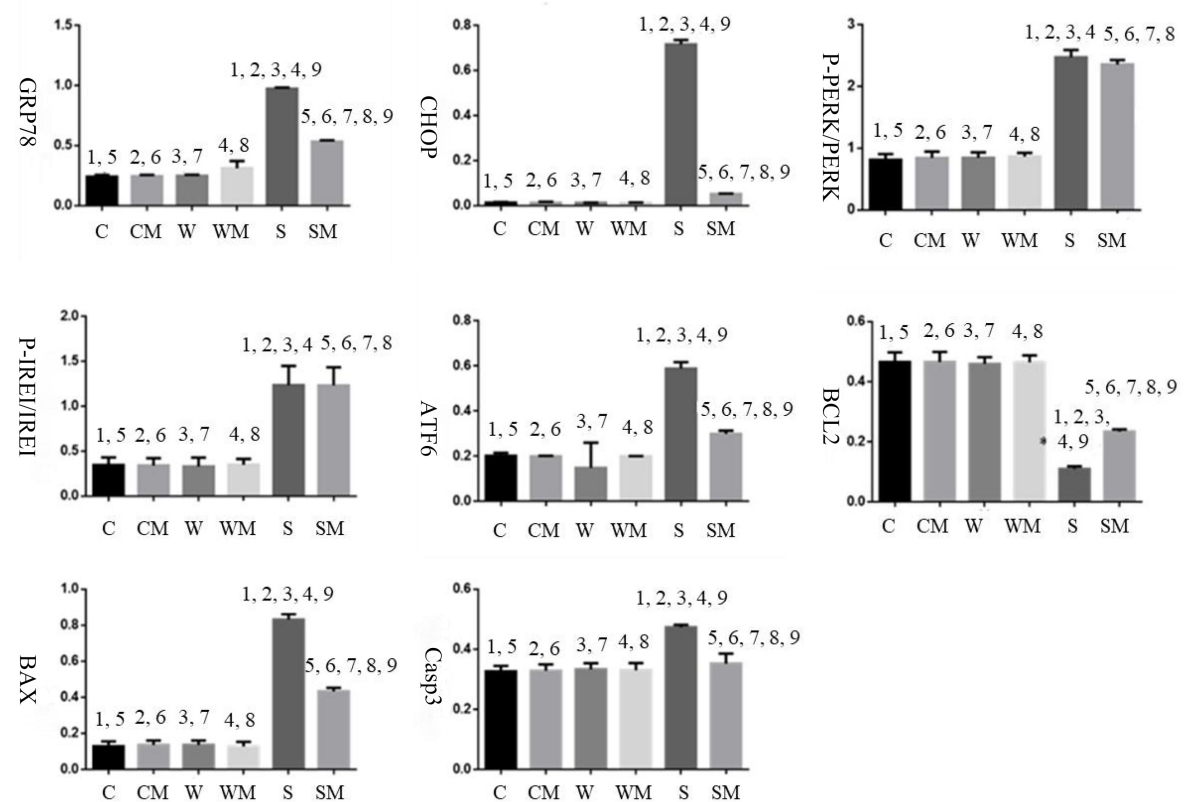

Fig. 7. GRP78, CHOP, ATF6, P-PERK/PERK, P-IRE1/IRE1, Bcl2, Bax, and Caspase3 expression in different groups. C: control, CM: control + MANF, W: wild type, WM: wild type + MANF, S: S63del MPZ, and SM: S63del MPZ + MANF. 1, 2, 3, 4, 5, 6, 7, 8, 9: The difference between two groups was statistically significant $(p<0.05) ; \mathrm{n}=3$.
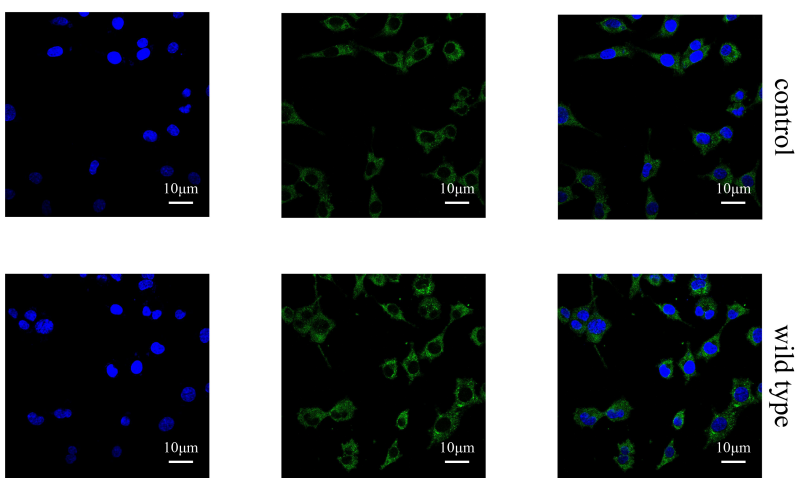

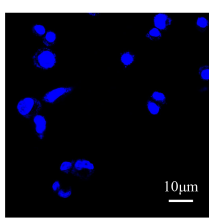

DAPI

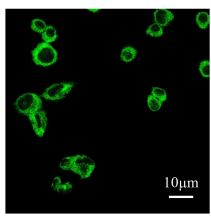

MANF

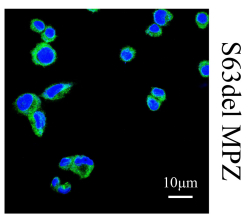

Merge
Fig. 8. MANF expression in each group.

For detecting protein levels, immunoblotting was performed. Briefly, samples were lysed in RIPA buffer containing $1 \mathrm{mM}$ PMSF. After centrifugation, 30-60 $\mu \mathrm{g}$ protein from each sample was loaded and transferred to a PVDF membrane. For immunofluorescence, cells were first washed with PBS and then fixed with $4 \%$ paraformaldehyde for $30 \mathrm{~min}$. Subsequently, the samples were incubated with the primary antibody at $4{ }^{\circ} \mathrm{C}$ overnight. Then, the samples were stained with a fluorophore-conjugated secondary

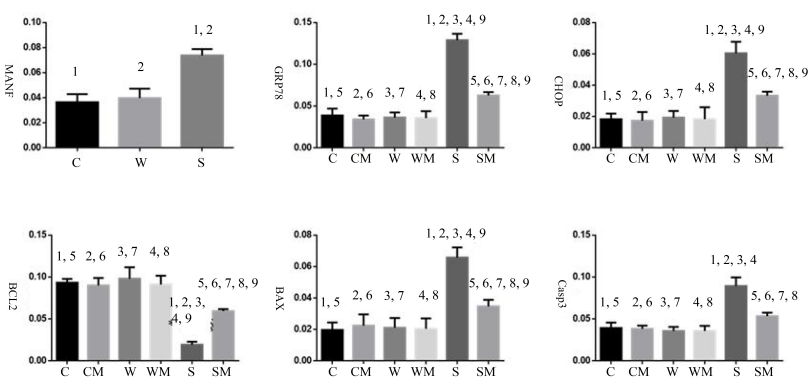

Fig. 9. MANF, GRP78, CHOP, Bcl2, Bax, and Caspase3 expression in each group. $\mathrm{C}$ : control, $\mathrm{CM}$ : control + MANF, W: wild type, WM: wild type + MANF, S: S63del MPZ, and SM: S63del MPZ + MANF.1, 2, 3, 4, 5, 6, 7, 8, 9: The difference between two groups was statistically significant $(p<0.05) ; \mathrm{n}=3$.

antibody for $30 \mathrm{~min}$. DAPI was used for nuclear staining. Images were acquired by confocal microscopy.

\subsection{ER-Tracker and Mito-Tracker dyes}

ER-Tracker Red (Beyotime, Shanghai, China) and Mito-Tracker Green (Beyotime, Shanghai, China) were used to observe the morphology of the ER and mitochondria. Images were acquired by using confocal microscopy.

\section{$2.9 \mathrm{Ca}^{+}$concentration of the ER}

The $\mathrm{Ca}^{+}$concentration in the ER was determined by using a GENMED Mag-Fluo-AM kit according to the manufacturer's protocol. 

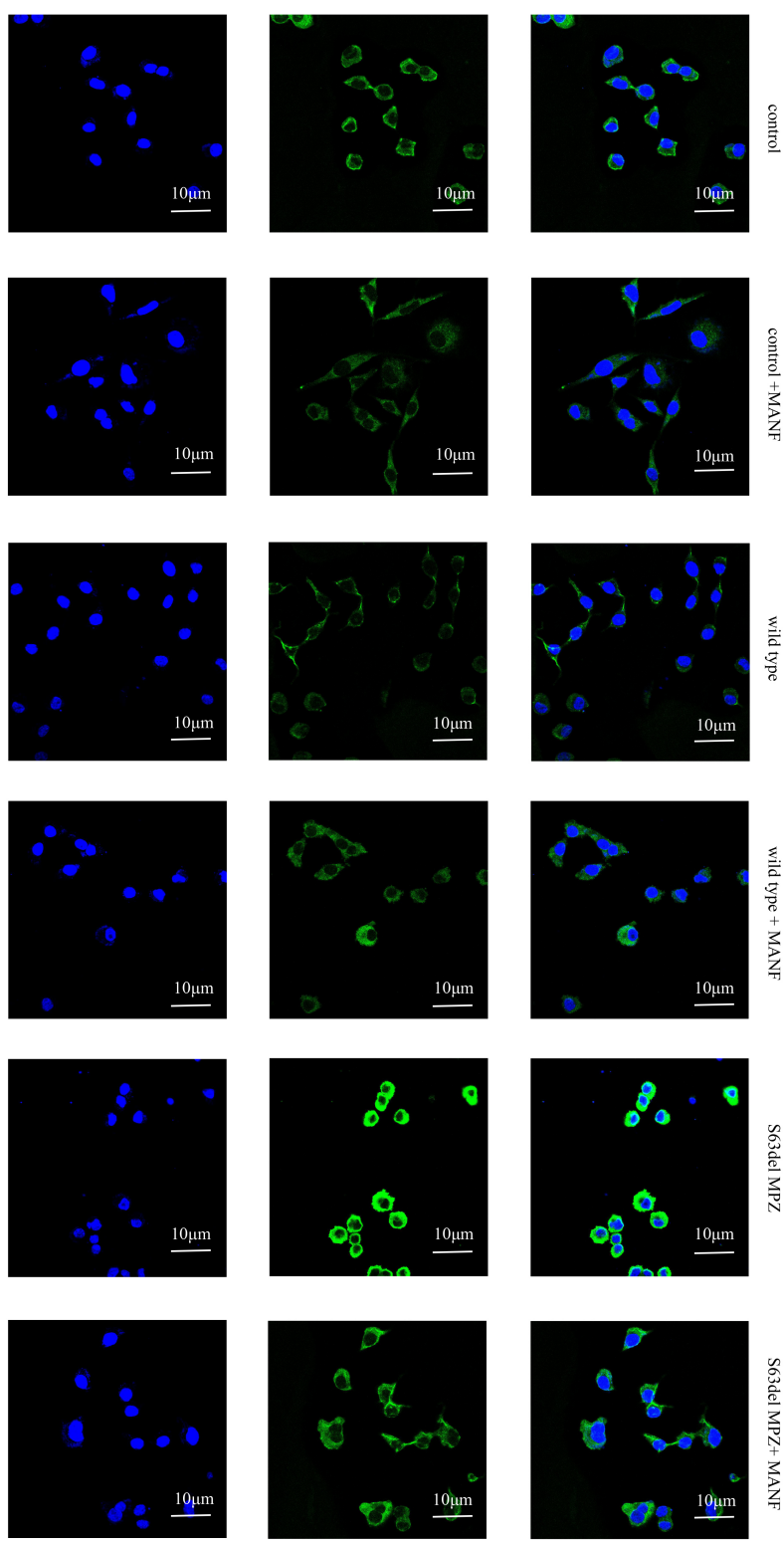

DAPI

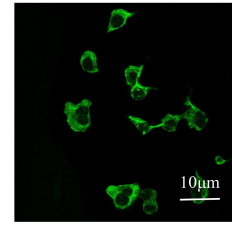

GRP78

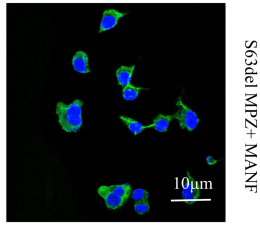

Merge

Fig. 10. GRP78 expression in each group.

\subsection{Statistical analysis}

Statistical analysis was performed using SPSS version 19.0 software (SPSS Inc., Chicago, IL, USA). Analysis of variance was performed to compare cell viability (CCK8), cell apoptosis (Annexin V-FITC/PI apoptosis detection), the $\triangle \Psi \mathrm{m}$, qPCR, Western blotting and immunofluorescence results of different groups. A two-tailed $p$-value $<$ 0.05 was considered statistically significant.

\section{Results}

\subsection{Cell morphology after 24 hours of culture}

After 24 hours of culture, the RT4-D6P2T schwannoma cells in each group completely adhered to the wall, and the cells stretched out pseudopods, showing irregular shapes. Cell morphology was similar within each group.
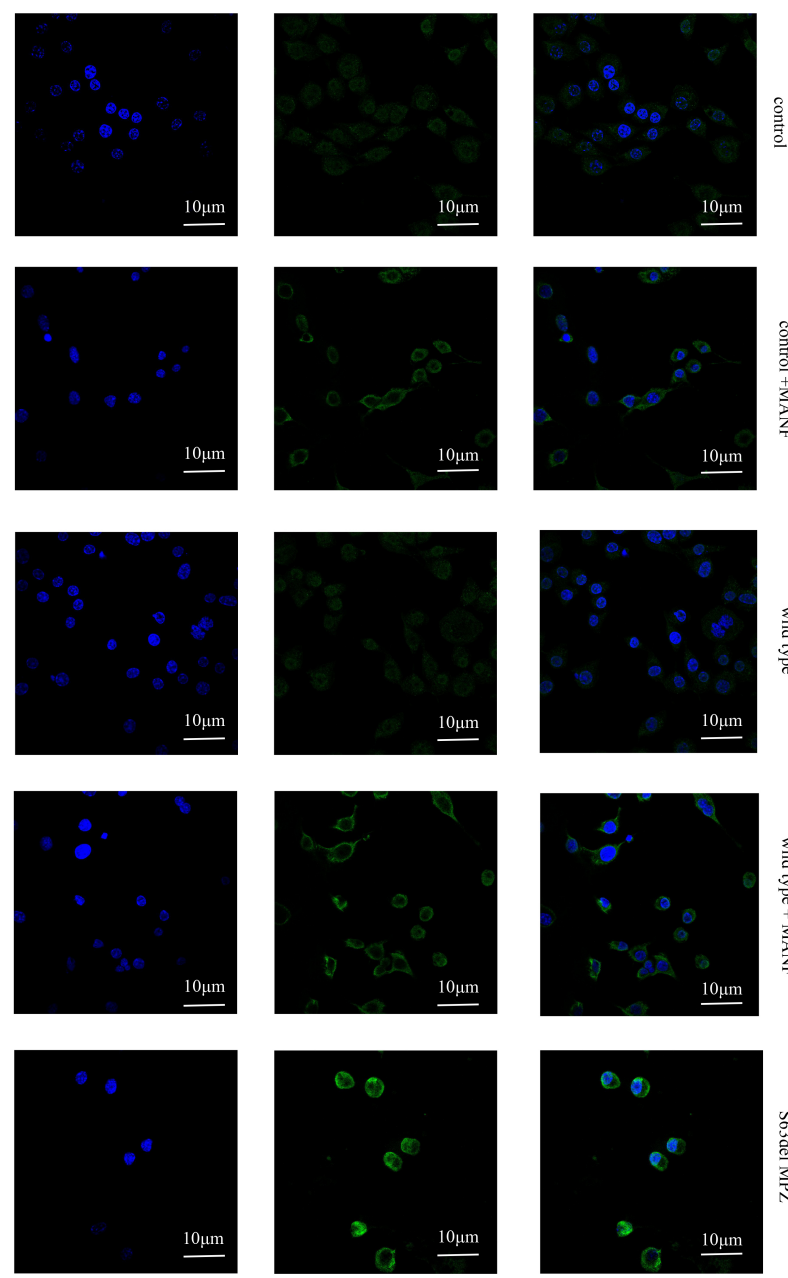

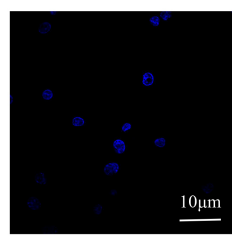

DAPI

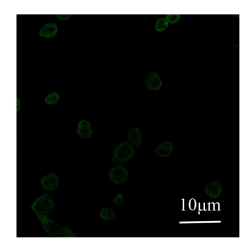

$\mathrm{CHOP}$

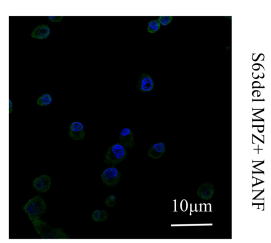

Merge
Fig. 11. CHOP expression in each group.

\subsection{CCK-8 cell proliferation and toxicity test}

CCK-8 was used for the cell proliferation and toxicity experiment. Variance analysis showed that there were significant differences among the groups $(\mathrm{F}=16.578, p<$ $0.001 ; \mathrm{n}=5$ ) (Table 1$)$. The optical density (OD) values of S63del MPZ and S63del MPZ + MANF groups were significantly increased compared with other groups (Table 1).

3.3 Flow cytometry: annexin V-FITC/PI double staining to evaluate apoptosis

When using annexin V-FITC/PI double staining to assess apoptosis, the upper left quadrant represents dead cells, the upper right quadrant represents late apoptotic cells, the lower left quadrant represents normal cells, and the lower right quadrant represents early apoptotic cells (Fig. 1). 

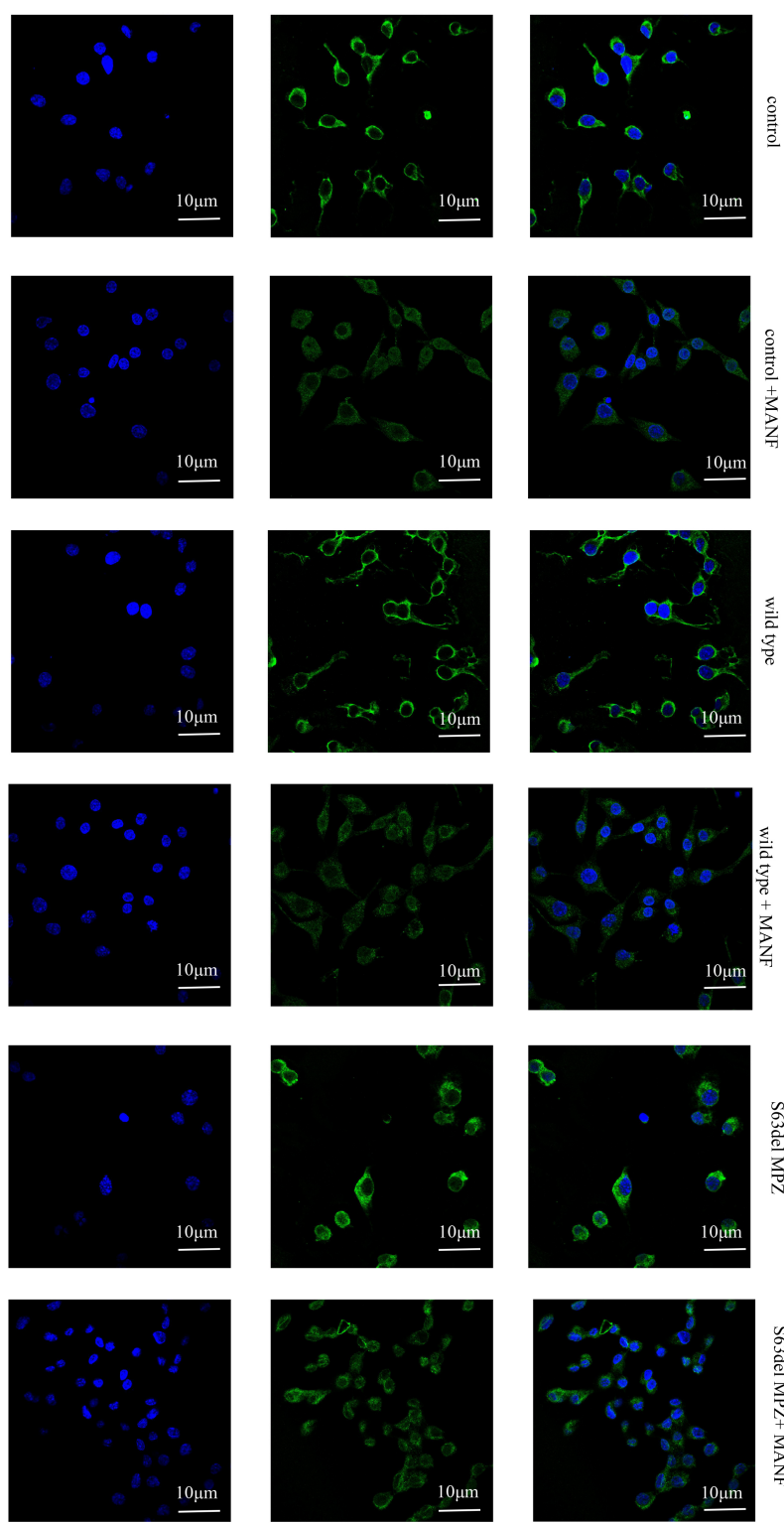

DAPI

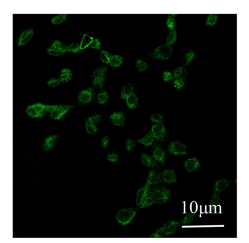

Bax

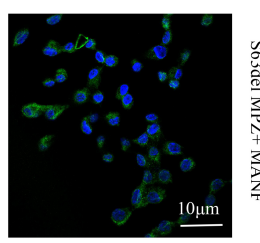

Merge

Fig. 12. Bax expression in each group.

There were significant differences among the groups $(\mathrm{F}=$ 65.419, $p<0.001 ; \mathrm{n}=3$ ) (Fig. 1). The ratio of apoptotic cells of S63del MPZ group was significantly increased than other groups (Fig. 1).

\subsection{Detection of the $\Delta \Psi m$}

The dye JC-1 has different forms at different $\Delta \Psi \mathrm{ms}$, thus producing fluorescence at different wavelengths, which can be measured by confocal microscopy or flow cytometry. Through confocal microscope observation (Fig. $2)$, the differences among the groups were significant $(\mathrm{F}=$ $18.271, p<0.001 ; \mathrm{n}=3$ ). The ratio of red to green fluorescence intensity of S63del MPZ group was significantly decreased compared with control $(p<0.001)$, control + MANF $(p<0.001)$, wild type $(p<0.001)$ and wild type
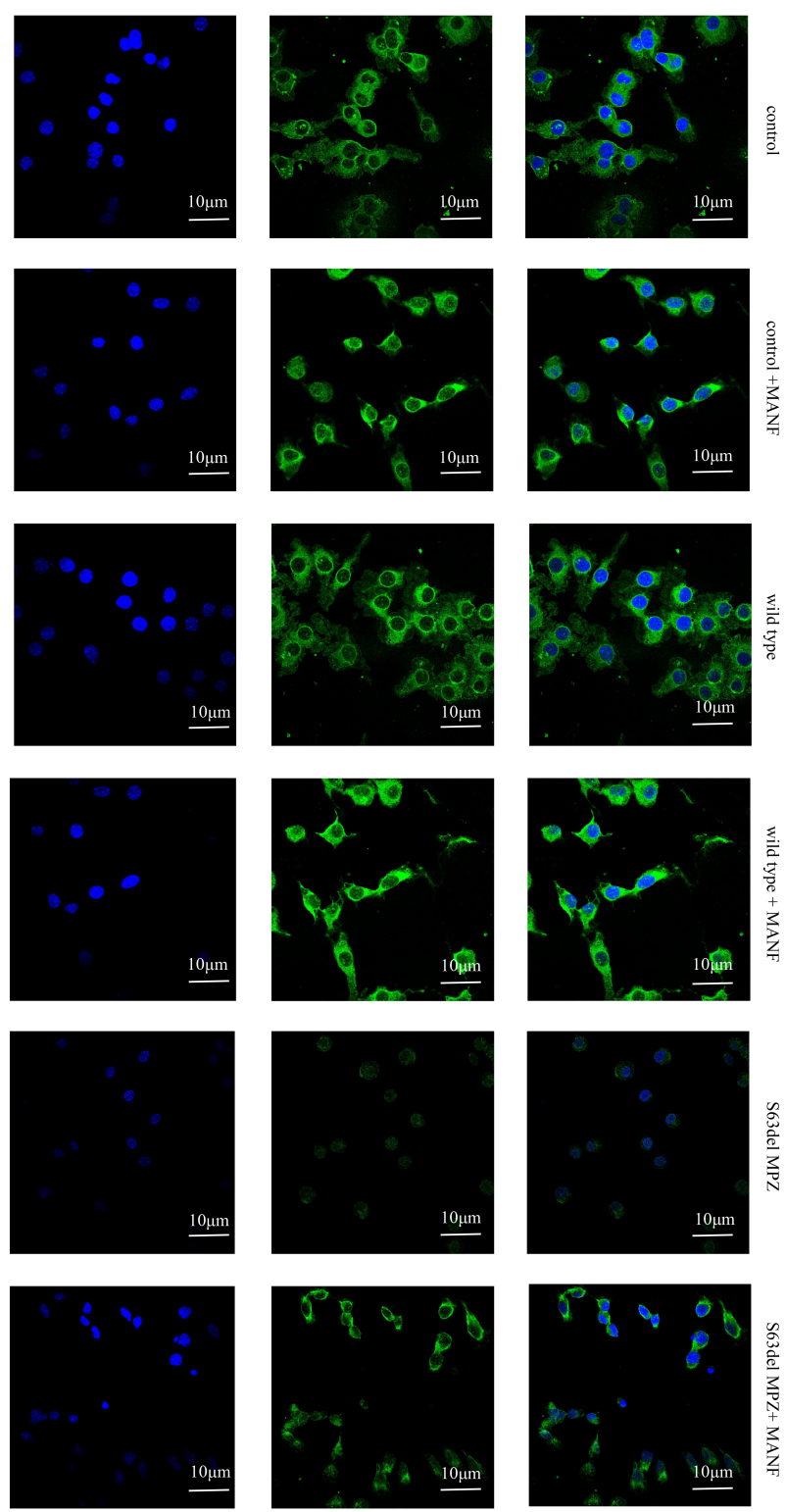

DAPI

$\operatorname{Bcl} 2$

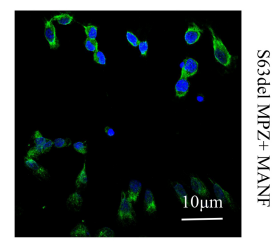

Merge

Fig. 13. Bcl2 expression in each group.

+ MANF group $(p<0.001)$ (Fig. 2).

In the process of flow cytometry detection, R2 represents apoptotic cells (Fig. 3), and the differences among the cells in different groups were significant $(\mathrm{F}=145.250, p<$ $0.001 ; n=3)$. The ratio of apoptotic cells of S63del MPZ group was significantly decreased compared with control $(p$ $<0.05)$, control + MANF $(p<0.05)$, wild type $(p<0.05)$ and wild type + MANF group $(p<0.05)$ (Fig. 3$)$.

3.5 Detection of the relative $m R N A$ expression of $M A N F$, GRP78 and CHOP in RT4-D6P2T cells

3.5.1 Detection of the relative mRNA expression of MANF

There was a significant difference in the relative mRNA expression of MANF among the cells in different 

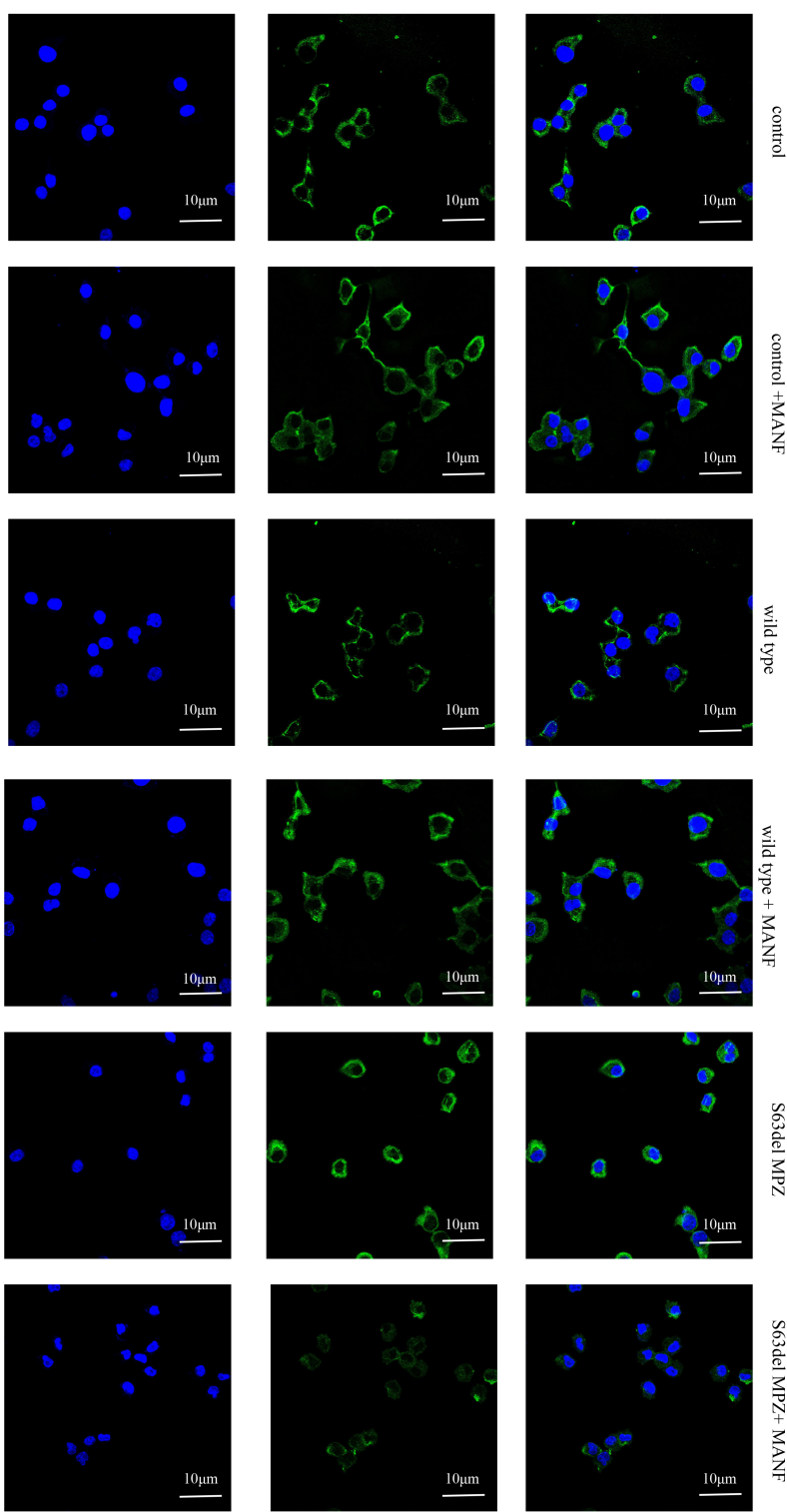

DAPI

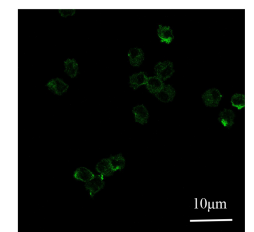

Caspase 3
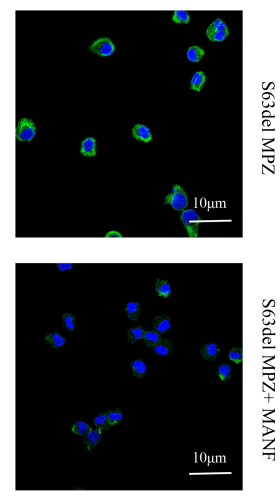

Merge

Fig. 14. Caspase3 expression in each group.
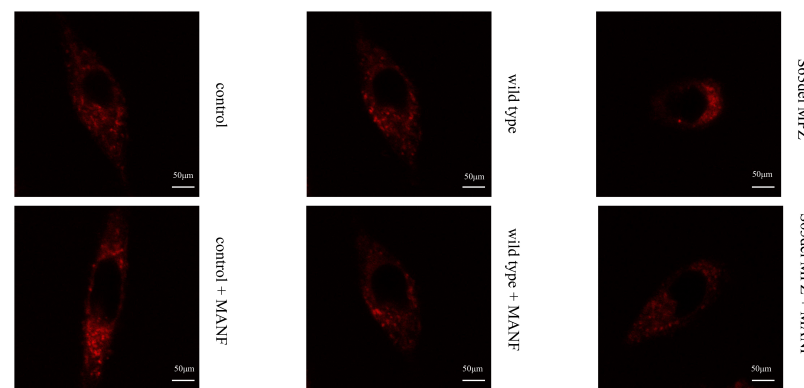

Fig. 15. ER-Tracker was used to detect the morphology of the endoplasmic reticulum.

groups $(\mathrm{F}=65.705, p<0.001 ; \mathrm{n}=3)$. The RQ value of S63del MPZ group was significantly increased compared
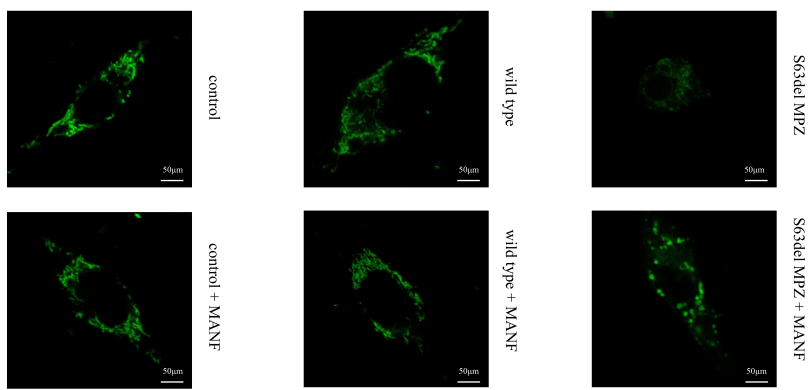

Fig. 16. Mito-Tracker was used to detect the morphology of the mitochondria.
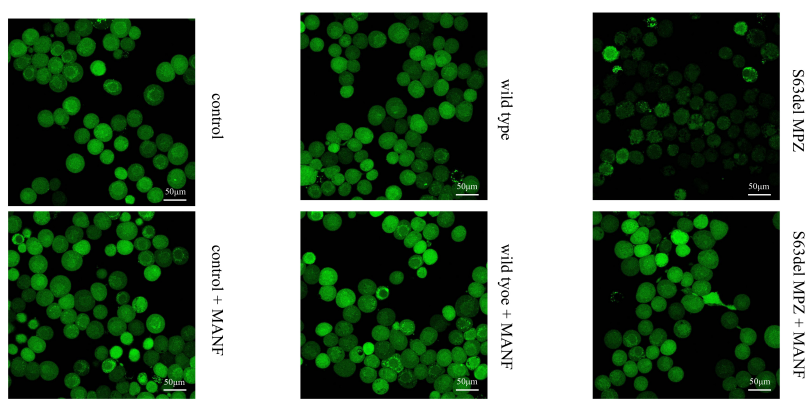

Fig. 17. Determination of the calcium ion concentration in the endoplasmic reticulum.

with control $(p<0.001)$ and wild type group $(p<0.001)$ (Table 2, Fig. 4).

\subsubsection{Detection of the relative mRNA expression of} GRP78

There were significant differences in the relative mRNA expression of GRP78 among the cells in different groups $(\mathrm{F}=718.26, p<0.001 ; \mathrm{n}=3)$. The RQ value of S63del MPZ group was significantly increased than other groups ( $p<0.001)$ (Table 3, Fig. 4).

\subsubsection{Detection of the relative mRNA expression of CHOP}

There were significant differences in the relative mRNA expression of CHOP among the cells in different groups $(\mathrm{F}=370.669, p<0.001 ; \mathrm{n}=3)$. The $\mathrm{RQ}$ value of S63del MPZ group was significantly increased than other groups $(p<0.001)$ (Table 4, Fig. 4).

\subsection{Western blot analysis}

(1) MANF: There were significant differences in MANF expression among the groups $(\mathrm{F}=95.245, p<$ $0.001, \mathrm{n}=3)$. MANF expression of S63del MPZ group was significantly increased $(p<0.001)$ (Fig. 5).

(2) GRP78, CHOP, ATF6, Bax and Caspase3: There were significant differences in GRP78 $(\mathrm{F}=363.593, p<$ $0.001 ; \mathrm{n}=3), \mathrm{CHOP}(\mathrm{F}=3210.661, p<0.001 ; \mathrm{n}=3)$, ATF6 $(\mathrm{F}=34.220, p<0.001 ; \mathrm{n}=3), \operatorname{Bax}(\mathrm{F}=385.522, p<0.001$; 
Table 1. CCK8 cell proliferation toxicity test.

\begin{tabular}{lcccc}
\hline OD & Mean & SD & F & $p$ \\
\hline Control $^{1,5}$ & 1.14 & 0.27 & 718.26 & 0 \\
Control + MANF $^{2,6}$ & 1.27 & 0.28 & & \\
Wild type $^{3,7}$ & 1.27 & 0.23 & & \\
Wild type + MANF group $^{4,8}$ & 1.21 & 0.20 & & \\
S63del MPZ $^{1,2,3,4}$ & 50.85 & 2.78 & & \\
S63del MPZ + MANF $^{5,6,7,8}$ & 40.29 & 2.33 & & \\
\hline 1,2,3,4,5,6,7,8 : significant differences $(p<0.05)$. & &
\end{tabular}

Table 2. MANF mRNA relative expression.

\begin{tabular}{lcccc}
\hline MANF & Mean & SD & F & $p$ \\
\hline Control $^{1}$ & 1.57 & 0.58 & 65.705 & 0 \\
Wild type $^{2}$ & 1.79 & 0.41 & & \\
S63del MPZ $^{1,2}$ & 20.37 & 3.93 & & \\
\hline 1,2 : significant differences $(p<0.001)$. &
\end{tabular}

$\mathrm{n}=3)$ and Caspase3 ( $\mathrm{F}=20.197, p<0.001 ; \mathrm{n}=3)$ expression among the groups. The expression of GRP78, CHOP, ATF6, Bax and Caspase 3 of S63del MPZ group was significantly increased than other groups $(p<0.001)$, including S63del MPZ + MANF group $(p<0.001)$ (Figs. 6,7).

(3) P-PERK/PERK and P-IRE1/IRE1: There were significant differences in P-PERK/PERK $(\mathrm{F}=228.797, p$ $<0.001 ; \mathrm{n}=3)$ and P-IRE1/IRE1 $(\mathrm{F}=33.440, p<0.001$; $\mathrm{n}=3$ ) expression among the groups. The expression of $\mathrm{P}-$ PERK/PERK and P-IRE1/IRE1 of S63del MPZ group was significantly increased than control $(p<0.001)$, control + MANF $(p<0.001)$, wild type $(p<0.001)$ and wild type + MANF groups $(p<0.001)$ (Figs. 6,7).

(4) Bcl2: There were significant differences in Bcl2 (F $=131.330, p<0.001 ; \mathrm{n}=3$ ) expression among the groups. The expression of Bcl2 of S63del MPZ group was significantly decreased than other groups $(p<0.001)$, including S63del MPZ + MANF group $(p<0.001)$ (Figs. 6,7).

3.7 The expression of MANF, GRP78, CHOP, Bcl2, Bax, and Caspase 3 evaluated by confocal microscopy

(1) MANF: There was a significant difference in the expression of MANF among the groups $(\mathrm{F}=30.731, p$ $<0.001, \mathrm{n}=3)$. MANF expression was significantly increased $(p<0.001)$ (Figs. 8,9).

(2) GRP78, CHOP and Bax: There were significant differences in GRP78 ( $\mathrm{F}=93.104, p<0.001 ; \mathrm{n}=3)$, CHOP $(\mathrm{F}=29.316, p<0.001 ; \mathrm{n}=3)$ and $\mathrm{Bax}(\mathrm{F}=26.986, p<$ $0.001 ; \mathrm{n}=3$ ) expression among the groups. The expression of GRP78, CHOP and Bax was significantly increased than other groups $(p<0.001)$, including S63del MPZ + MANF group $(p<0.001)$ (Figs. 9, 10,11,12).

(3) Bcl2: There were significant differences in $\mathrm{Bcl} 2$ (F $=41.082, p<0.001 ; \mathrm{n}=3$ ) expression among the groups. The expression of Bcl 2 was significantly decreased than
Table 3. GRP78 mRNA relative expression.

\begin{tabular}{lcccc}
\hline GRP78 & Mean & SD & F & $p$ \\
\hline Control $^{1,5}$ & 1.14 & 0.27 & 718.26 & 0 \\
Control + MANF $^{2,6}$ & 1.27 & 0.28 & & \\
Wild type $^{3,7}$ & 1.27 & 0.23 & & \\
Wild type + MANF $^{4,8}$ & 1.21 & 0.2 & & \\
S63del MPZ $^{1,2,3,4,9}$ & 50.85 & 2.78 & & \\
S63del MPZ + MANF $^{5,6,7,8,9}$ & 40.29 & 2.33 & & \\
\hline $1,2,3,4,5,6,7,8,9$ & : significant differences $(p<0.001)$.
\end{tabular}

Table 4. CHOP mRNA relative expression.

\begin{tabular}{lcccc}
\hline CHOP & Mean & SD & F & $p$ \\
\hline Control $^{1,5}$ & 1.36 & 0.31 & 370.669 & 0 \\
Control + MANF $^{2,6}$ & 1.5 & 0.13 & & \\
Wild type $^{3,7}$ & 1.28 & 0.24 & & \\
Wild type + MANF $^{4,8}$ & 1.3 & 0.19 & & \\
S63del MPZ $^{1,2,3,4,9}$ & 24.73 & 2.09 & & \\
S63del MPZ + MANF $^{5,6,7,8,9}$ & 16.91 & 0.78 & & \\
\hline
\end{tabular}

$1,2,3,4,5,6,7,8,9$ : significant differences $(p<0.001)$.

other groups $(p<0.001)$, including S63del MPZ + MANF group $(p<0.001)$ (Figs. 9,13).

(4) Caspase3: There were significant differences in the expression of Caspase 3 among the groups $(\mathrm{F}=33.263$, $p<0.001 ; \mathrm{n}=3)$. The expression of Caspase3 of S63del MPZ group was significantly increased than control $(p<$ $0.001)$, control + MANF $(p<0.001)$, wild type $(p<0.001)$ and wild type + MANF groups $(p<0.001)$ (Figs. 9,14).

\subsection{ER-Tracker staining}

An ER-Tracker dye was used to detect the morphology of the ER in each group. It was found that in the S63del MPZ group, the ER lost its original network structure and the structure was unclear. In the S63del MPZ + MANF group, this phenomenon was improved (Fig. 15).

\subsection{Mito-Tracker staining}

Mito-Tracker was used to detect the morphology of the mitochondria in each group. It was found that in the S63del MPZ group, the mitochondria lost their original reticular structure, the staining was not clear, and the structure was unclear. This phenomenon was improved in the S63del MPZ + MANF group compared with the S63del MPZ group (Fig. 16).

\subsection{Determination of the calcium ion concentration in the ER}

The results showed that the calcium concentration in the ER was decreased in the S63del MPZ group and improved in the S63del MPZ + MANF group (Fig. 17). 


\section{Discussion}

ERS has been observed in a CMT1B mouse model (S63del MPZ and R98C MPZ mutations) [6-8]. To date, there have been no studies of related cell models. In this study, RT4-D6P2T schwannoma cells stably transfected with the S63del MPZ mutation were established to investigate whether ERS occurs in RT4-D6P2T cells with the S63del MPZ mutation.

With the accumulation of large amounts of unfolded proteins, ER homeostasis is disrupted, which leads to the dissociation of PERK, ATF6 and IRE1 from GRP78, initiating three major signal transduction pathways of ERS [11,32-34]. Upregulation of GRP78 expression promotes protein folding and restores ER homeostasis. Under ERS conditions, activated PERK and ATF6 induce the expression of CHOP, which is very low under physiological conditions. CHOP is the marker protein for ERS. In this study, the upregulation of p-PERK/PERK, p-IRE1/IRE1, ATF6, GRP78 and CHOP expression; morphological changes in the ER revealed by the ER-Tracker dye; and reduced concentration of $\mathrm{Ca}^{+}$in the ER hinted that protein overexpression overloading the ER leading to ERS occurred in RT4D6P2T cells with the S63del MPZ mutation.

MANF is a recently discovered $18-\mathrm{kD}$ soluble protein that is located in the ER lumen, and its expression is upregulated under ERS. MANF mRNA and protein are widely expressed in neuronal and nonneuronal tissues [28]. In recent years, in vivo and in vitro experiments have indicated that MANF exerts protective effects on ERS [11,29-36]. In the U2OS, HEK293, SH-SY5Y and NIH3T3 cell lines, MANF upregulation is detected during ERS induced by tunicamycin, carotene and lactomycin [29,30]. MANF upregulation is also detected during ERS in a chondrodysplasia mouse model [31]. ERS also occurs in a diabetic mouse model with the $\mathrm{C} 96 \mathrm{Y}$ mutation and in a kidney disease mouse model with the C321R mutation, under which conditions MANF expression is upregulated [29]. In addition, MANF upregulation has been detected in in vivo and in vitro ischemic experiments [33-36]. In our study, ERS occurred in RT4-D6P2T cells with the S63del MPZ mutation, and MANF mRNA and protein levels were upregulated, which was consistent with previous studies.

The upregulation of ERS-related MANF expression is realized by ERS response element (ERSE)-II located in the promoter region of the MANF gene [29,35]. ERSEII (ACGTGGNCCAAT) contains two transcription factor recognition sequences: ACGTGG, which is recognized by ATF6 and XBP1, and CCAAT, which is recognized by NFY. In Neuro2a cells, when ERS occurs, ATF6a promotes the activity of the MANF promoter and the transcription of MANF by combining with ERSE-II. However, XBP1 exerts a relatively weak function [37]. Furthermore, increased expression of MANF has also been detected in the cardiomyocytes of ATF6 transgenic mice, suggesting that ATF6 promotes the expression of MANF [38]. Thus, in our research, the increased mRNA and protein expression of MANF is probably due to the combination of ATF6 and ERSE-II to promote the transcription of MANF.

Exogenous MANF plays a protective role in an ERSrelated disease model. In vitro, recombinant human MANF intervention promotes the growth of islet $\beta$ cells. In addition, in a diabetic mouse model, overexpression of MANF in the pancreas promotes the regeneration of islet $\beta$ cells [39], suggesting that MANF may play an important role in the treatment of diabetes. MANF also plays important roles in neurodegenerative diseases, such as Parkinson's disease. In vitro, MANF exerts a protective effect on dopaminergic neurons [22]. In Drosophila, MANF deficiency leads to the underdevelopment of dopaminergic neurons, which develop normally after supplementation with MANF [40]. In the 6-OHDA-induced Parkinson's disease model in rats, injection of MANF promotes the functional recovery of the dopamine system in the striatum nigra, which may be related to the inhibition of ERS by MANF [41]. Furthermore, MANF is also abundantly expressed in Purkinje cells [42]. In the SCA17 mouse model, the expression of MANF in the cerebellum is decreased, while MANF overexpression mediated by a lentivirus reduces the loss of Purkinje cells and alleviates ataxia [43]. In this study, ERS occurred in RT4-D6P2T cells with the S63del MPZ mutation. GRP78, CHOP and ATF6 levels were downregulated, ER morphology was improved, the calcium concentration in the ER was restored, and apoptosis was decreased with MANF intervention, suggesting that MANF may inhibit ERS, which is consistent with previous studies [22,39-43].

In addition, the mitochondria play an important role in ERS-induced apoptosis. There are abundant studies on the molecular mechanism of apoptosis in ERS, but few reports have mentioned mitochondrial damage in ERS [44]. The ER and mitochondria are two important organelles related to each other in cell function. They interact with each other through a variety of proteins to complete biological functions. For example, under ERS conditions, GRP78 translocates into the mitochondria, which may inhibit the decrease in the $\Delta \Psi \mathrm{m}$ induced by ERS [45-47]. In this study, ERS occurred in RT4-D6P2T cells with the S63del MPZ mutation, and mitochondrial metabolic activities related to apoptosis changed: Bcl-2 expression decreased, Bax and caspase- 3 expression increased, and the $\Delta \Psi \mathrm{m}$ decreased. Mito-Tracker staining suggested that the mitochondrial structure was damaged and apoptosis increased. There are three classic pathways of cell apoptosis, among which the mitochondrial pathway plays an important role. On the one hand, cytochrome $\mathrm{C}$ in the mitochondrial membrane space is released into the cytoplasm and triggers the caspase cascade, which leads to cell apoptosis; on the other hand, the bcl-2 protein family expressed on the mitochondrial outer membrane regulates the release of cytochrome $\mathrm{C}$ from the mitochondria. When apoptosis occurs, the mitochondria undergo a series of changes, including mitochon- 
drial fragmentation, $\Delta \Psi \mathrm{m}$ loss and mitochondrial respiratory function damage. These changes are important markers of the mitochondrial apoptosis pathway [48,49], which is consistent with the mitochondrial functional damage observed in this study.

The findings of the study require confirmation by further experiments in vivo, that is, in a CMT1B mouse model with the S63del MPZ mutation. Further studies should be conducted to determine whether XBP1 and NF-Y play a role in this process. Signal pathway inhibitors can be added to clarify which signaling pathway MANF acts through to play a protective role in ERS in RT4-D6P2T cells with the S63del MPZ mutation.

\section{Conclusions}

This study found that ERS occurred in RT4-D6P2T cells with the S63del MPZ mutation and MANF exerted a protective effect on the RT4-D6P2T cells with the S63del MPZ mutation, which requires further confirmation in vivo.

\section{Author contributions}

BS and XSH designed the study. BS, HFW, YRL and $\mathrm{ZQH}$ conducted the study. BS, FY and FC collected and analysed the data. BS drafted and wrote the manuscript. XSH revised the manuscript critically for intellectual content. All authors gave intellectual input to the study and approved the final version of the manuscript.

\section{Ethics approval and consent to participate}

This study was approved by the Ethics Committee of Chinese PLA General Hospital (S2016-021-01).

\section{Acknowledgment}

We would like to thank our colleagues for their contributions to this research work.

\section{Funding}

This research was funded by the National Natural Science Foundation of China to Xusheng Huang, grant number 81870989 and to Bo Sun, grant number 81901274 .

\section{Conflict of interest}

The authors declare no conflict of interest.

\section{References}

[1] Tazir M, Hamadouche T, Nouioua S, Mathis S, Vallat JM. Hereditary motor and sensory neuropathies or Charcot-MarieTooth diseases: an update. Journal of the neurological sciences. 2014; 347: 14-22.

[2] Zhao HT, Damle S, Ikeda-Lee K, Kuntz S, Li J, Mohan A, et al. PMP22 antisense oligonucleotides reverse Charcot-Marie-Tooth disease type $1 \mathrm{a}$ features in rodent models. The Journal of Clinical Investigation. 2018; 128: 359-368.

[3] Nelis E, Van Broeckhoven C, De Jonghe P, Löfgren A, Vandenberghe A, Latour $\mathrm{P}$, et al. Estimation of the mutation frequencies in Charcot-Marie-Tooth disease type 1 and hereditary neuropathy with liability to pressure palsies: a European collaborative study. European Journal of Human Genetics. 1996; 4: 25-33.

[4] Saporta AS, Sottile SL, Miller LJ, Feely SM, Siskind CE, Shy ME. Charcot-Marie-Tooth disease subtypes and genetic testing strategies. Annals of Neurology. 2011; 69: 22-33.

[5] Timmerman V, Strickland AV, Züchner S. Genetics of CharcotMarie-Tooth (CMT) Disease within the Frame of the Human Genome Project Success. Genes. 2014; 5: 13-32.

[6] Sidoli M, Musner N, Silvestri N, Ungaro D, D’Antonio M, Cavener DR, et al. Ablation of Perk in Schwann Cells Improves Myelination in the S63del Charcot-Marie-Tooth 1B Mouse. The Journal of Neuroscience. 2016; 36: 11350-11361.

[7] Pennuto M, Tinelli E, Malaguti M, Del Carro U, D’Antonio M, Ron D, et al. Ablation of the UPR-mediator CHOP restores motor function and reduces demyelination in Charcot-Marie-Tooth 1B mice. Neuron. 2008; 57: 393-405.

[8] Patzkó A, Bai Y, Saporta MA, Katona I, Wu X, Vizzuso D, et al. Curcumin derivatives promote Schwann cell differentiation and improve neuropathy in R98C CMT1B mice. Brain: a Journal of Neurology. 2012; 135: 3551-3566.

[9] D’Antonio M, Musner N, Scapin C, Ungaro D, Del Carro U, Ron $\mathrm{D}$, et al. Resetting translational homeostasis restores myelination in Charcot-Marie-Tooth disease type 1B mice. The Journal of Experimental Medicine. 2013; 210: 821-838.

[10] Schröder M, Kaufman RJ. The mammalian unfolded protein response. Annual Review of Biochemistry. 2005; 74: 739-789.

[11] Walter P, Ron D. The unfolded protein response: from stress pathway to homeostatic regulation. Science. 2011; 334: 10811086.

[12] Tabas I, Ron D. Integrating the mechanisms of apoptosis induced by endoplasmic reticulum stress. Nature Cell Biology. 2011; 13: 184-190.

[13] Bertolotti A, Ron D. Alterations in an IRE1-RNA complex in the mammalian unfolded protein response. Journal of Cell Science. 2001; 114: 3207-3212.

[14] Calfon M, Zeng H, Urano F, Till JH, Hubbard SR, Harding HP, et al. IRE1 couples endoplasmic reticulum load to secretory capacity by processing the XBP-1 mRNA. Nature. 2002; 415: 92 96.

[15] Yoshida H, Matsui T, Yamamoto A, Okada T, Mori K. XBP1 mRNA is induced by ATF6 and spliced by IRE1 in response to ER stress to produce a highly active transcription factor. Cell. 2001; 107: 881-891.

[16] Lee A, Iwakoshi NN, Glimcher LH. XBP-1 regulates a subset of endoplasmic reticulum resident chaperone genes in the unfolded protein response. Molecular and Cellular Biology. 2003; 23: 7448-7459.

[17] Yoshida H, Matsui T, Hosokawa N, Kaufman RJ, Nagata K, Mori K. A time-dependent phase shift in the mammalian unfolded protein response. Developmental Cell. 2003; 4: 265-271.

[18] Sriburi R, Jackowski S, Mori K, Brewer JW. XBP1: a link between the unfolded protein response, lipid biosynthesis, and biogenesis of the endoplasmic reticulum. The Journal of Cell Biology. 2004; 167: 35-41.

[19] Shen J, Chen X, Hendershot L, Prywes R. ER stress regulation of ATF6 localization by dissociation of BiP/GRP78 binding and unmasking of Golgi localization signals. Developmental Cell. 2002; 3: 99-111.

[20] Harding HP, Zhang Y, Bertolotti A, Zeng H, Ron D. Perk is essential for translational regulation and cell survival during the unfolded protein response. Molecular Cell. 2000; 5: 897-904.

[21] Das I, Krzyzosiak A, Schneider K, Wrabetz L, D’Antonio M, Barry $\mathrm{N}$, et al. Preventing proteostasis diseases by selective inhibition of a phosphatase regulatory subunit. Science. 2015; 348: 239-242. 
[22] Petrova P, Raibekas A, Pevsner J, Vigo N, Anafi M, Moore MK, et al. MANF: a new mesencephalic, astrocyte-derived neurotrophic factor with selectivity for dopaminergic neurons. Journal of Molecular Neuroscience. 2003; 20: 173-188.

[23] Lindahl M, Saarma M, Lindholm P. Unconventional neurotrophic factors CDNF and MANF: structure, physiological functions and therapeutic potential. Neurobiology of Disease. 2017; 97: 90-102.

[24] Lindahl M, Danilova T, Palm E, Lindholm P, Võikar V, Hakonen $\mathrm{E}$, et al. MANF is indispensable for the proliferation and survival of pancreatic beta cells. Cell Reports. 2014, 7:366-375.

[25] Wei S, Yu Y, Weiss RM, Felder RB. Endoplasmic reticulum stress increases brain MAPK signaling, inflammation and reninangiotensin system activity and sympathetic nerve activity in heart failure. American Journal of Physiology-Heart and Circulatory Physiology. 2016; 311: H871-H880.

[26] Jeong K, Oh Y, Kim S, Kim H, Park K, Kim SS, et al. Apelin is transcriptionally regulated by ER stress-induced ATF4 expression via a p38 MAPK-dependent pathway. Apoptosis. 2014; 19: 1399-1410.

[27] Cubillos-Ruiz JR, Silberman PC, Rutkowski MR, Chopra S, Perales-Puchalt A, Song M, et al. ER stress sensor XBP1 controls anti-tumor immunity by disrupting Dendritic cell homeostasis. Cell. 2015, 161:1527-1538.

[28] Sun H, Jiang M, Fu X, Cai Q, Zhang J, Yin Y, et al. Mesencephalic astrocyte-derived neurotrophic factor reduces cell apoptosis via upregulating HSP70 in SHSY-5Y cells. Translational Neurodegeneration. 2017; 6: 12.

[29] Wu T, Zhang F, Yang Q, Zhang Y, Liu Q, Jiang W, et al. Circulating mesencephalic astrocyte-derived neurotrophic factor is increased in newly diagnosed prediabetic and diabetic patients, and is associated with insulin resistance. Endocrine Journal. 2017; 64: 403-410.

[30] Kim Y, Lee H, Manson SR, Lindahl M, Evans B, Miner JH, et al. Mesencephalic Astrocyte-Derived Neurotrophic Factor as a Urine Biomarker for Endoplasmic Reticulum Stress-Related Kidney Diseases. Journal of the American Society of Nephrology. 2016; 27: 2974-2982.

[31] Voutilainen MH, Arumäe U, Airavaara M, Saarma M. Therapeutic potential of the endoplasmic reticulum located and secreted CDNF/MANF family of neurotrophic factors in Parkinson's disease. FEBS Letters. 2015; 589: 3739-3748.

[32] Stone $\mathrm{S}$, Lin W. The unfolded protein response in multiple sclerosis. Frontiers in Neuroscience. 2015; 9: 264.

[33] Oslowski CM, Urano F. Measuring ER stress and the unfolded protein response using mammalian tissue culture system. Methods in Enzymology. 2011; 490: 71-92.

[34] Ron D, Walter P. Signal integration in the endoplasmic reticulum unfolded protein response. Nature Reviews. Molecular Cell Biology. 2007; 8: 519-529.

[35] Tadimalla A, Belmont PJ, Thuerauf DJ, Glassy MS, Martindale JJ, Gude N, et al. Mesencephalic astrocyte-derived neurotrophic factor is an ischemia-inducible secreted endoplasmic reticulum stress response protein in the heart. Circulation Research. 2008; 103: 1249-1258.

[36] Yu Y, Liu L, Wang F, Liang Y, Cha D, Zhang J, et al. Induction profile of MANF/ARMET by cerebral ischemia and its implication for neuron protection. Journal of Cerebral Blood Flow \& Metabolism. 2010; 30: 79-91.

[37] Oh-Hashi K, Hirata Y, Kiuchi K. Transcriptional regulation of mouse mesencephalic astrocyte-derived neurotrophic factor in Neuro2a cells. Cellular \& Molecular Biology Letters. 2013; 18: 398-415.

[38] Martindale JJ, Fernandez R, Thuerauf D, Whittaker R, Gude $\mathrm{N}$, Sussman MA, et al. Endoplasmic reticulum stress gene induction and protection from ischemia/reperfusion injury in the hearts of transgenic mice with a tamoxifen-regulated form of ATF6. Circulation Research. 2006; 98: 1186-1193.

[39] Lindahl M, Danilova T, Palm E, Lindholm P, Võikar V, Hakonen $\mathrm{E}$, et al. MANF is indispensable for the proliferation and survival of pancreatic beta cells. Cell Reports. 2014; 7: 366-375.

[40] Palgi M, Lindström R, Peränen J, Piepponen TP, Saarma M, Heino TI. Evidence that DmMANF is an invertebrate neurotrophic factor supporting dopaminergic neurons. Proceedings of the National Academy of Sciences of the United States of America. 2009; 106: 2429-2434.

[41] Voutilainen MH, Back S, Porsti E, Toppinen L, Lindgren $\mathrm{L}$, Lindholm $\mathrm{P}$, et al. Mesencephalic astrocyte-derived neurotrophic factor is neurorestorative in rat model of Parkinson's Disease. Journal of Neuroscience. 2009; 29: 9651-9659.

[42] Lindholm P, Peränen J, Andressoo J, Kalkkinen N, Kokaia Z, Lindvall $\mathrm{O}$, et al. MANF is widely expressed in mammalian tissues and differently regulated after ischemic and epileptic insults in rodent brain. Molecular and Cellular Neurosciences. 2008; 39: $356-371$.

[43] Yang S, Huang S, Gaertig M, Li X, Li S. Age-Dependent Decrease in Chaperone Activity Impairs MANF Expression, Leading to Purkinje Cell Degeneration in Inducible SCA17 Mice. Neuron. 2014; 81: 349-365.

[44] Bravo R, Gutierrez T, Paredes F, Gatica D, Rodriguez AE, Pedrozo Z, et al. Endoplasmic reticulum: ER stress regulates mitochondrial bioenergetics. The International Journal of Biochemistry \& Cell Biology. 2012; 44: 16-20.

[45] Senft D, Ronai ZA. UPR, autophagy, and mitochondria crosstalk underlies the ER stress response. Trends in Biochemical Sciences. 2015; 40: 141-148.

[46] Shi J, Jiang Q, Ding X, Xu W, Wang DW, Chen M. The ER stress-mediated mitochondrial apoptotic pathway and MAPKs modulate tachypacing-induced apoptosis in HL-1 atrial myocytes. PLoS ONE. 2015; 10: e0117567.

[47] Deegan S, Saveljeva S, Logue SE, Pakos-Zebrucka K, Gupta S, Vandenabeele $\mathrm{P}$, et al. Deficiency in the mitochondrial apoptotic pathway reveals the toxic potential of autophagy under ER stress conditions. Autophagy. 2014; 10: 1921-1936.

[48] Contreras L, Drago I, Zampese E, Pozzan T. Mitochondria: the calcium connection. Biochimica et Biophysica Acta (BBA)Bioenergetics. 2010; 1797: 607-618.

[49] Moreira AC, Branco AF, Sampaio SF, Cunha-Oliveira T, Martins TR, Holy J, et al. Mitochondrial apoptosis-inducing factor is involved in doxorubicin-induced toxicity on H9c2 cardiomyoblasts. Biochimica et Biophysica Acta (BBA)-Molecular Basis of Disease. 2014; 1842: 2468-2478. 